\title{
Being together - Exploring the modulation of affect in improvisational music therapy with a man in a persistent vegetative state - a qualitative single case study
}

This article explores the role of affective expression and modulation as a means of communication in improvisational music therapy with a 44-year-old man living in a persistent vegetative state. Within a practice-based approach two vignettes from music therapy illustrate the regulation of the intensity of affect in an interpersonal relationship. Perspectives from modern attachment theory, developmental psychology, and embodiment research will be introduced and discussed, to theoretically frame and embed the practical work. It is suggested that the bodily- emotional situatedness of the man and the music therapist form the area of exchange for a non-verbal, affect-driven communication. In this way, playing with the affect is the main topic for the encounter, promoting self-organizational processes in both individuals involved.

\section{KEY WORDS}

improvisational music therapy; affect modulation; embodiment; isolation; neurological trauma 


\section{AFFECT - A MULTIFACETED PHENOMENON}

Affect regulation is one important aspect of self-regulation in a human being. It is vital for both individual well-being and social interaction (Barrett, Fox, Morgan, Fidler, \& Daunhauer, 2013). One approach to an understanding of affect regulation processes is attachment theory, developed by John Bowlby in the late 1960s. He suggested that human beings are born with an innate psychobiological system, which motivates them to seek proximity to significant others, called attachment figures, to get protection from physical and psychological threats and to alleviate stress in times of need (Bowlby, 1969/1982, 1988). The basic concept underlying these processes is the development of intersubjectivity - human beings' quest for continuous and reciprocal interaction and exchange from the very first days of their life (Ammaniti \& Gallese, 2014). Attachment figures provide a secure base from which individuals can explore and learn about the world and develop their capabilities and personalities. Nowadays attachment theory is seen as one of the most useful and generative frameworks to understand affect regulation (Mikulincer, Shaver, \& Pereg, 2003). Attachment styles begin to develop during early childhood in interactions with primary caregivers, and can change, subtly or dramatically, depending on the current context and recent relational experience over a lifespan (Shaver \& Mikulincer, 2014). Schore and Schore (2008) state that within a shared culture and environment the mother initially provides an external regulating mechanism for many of the physiological mechanisms that the infant possesses, but is not yet able to regulate on his or her own. Over the course of infancy, a child becomes increasingly self-regulated as a result of neurophysiological development. In this way individuals adapt the ability to flexibly regulate psychobiological states of emotions through interactions with other humans, as well as on their own. The more a mother contingently tunes her activity level to the infant during periods of social engagement, the more she allows him to recover in periods of disengagement, and the more she attends to his reinitiating cues for reengagement, the more synchronized their interaction. "In play episodes of affect synchrony, the pair are in affective resonance, and in such, an amplification of vitality affects and a positive state occurs" (Schore \& Schore, 2008, p. 11). On a preverbal matrix sequences of attunement, mis-attunement, and re-attunement, as well as the transitions from one mode to the other are important for human self-regulation and well-being. On this background Fuchs and De Jaegher (2009) suggest that it is the coordination of two embodied subjects mutually influencing each other that leads to an emotionally shared activity, evoking corresponding feelings and gradual development of affective resonance. They present a concept of enactive intersubjectivity that relies heavily on dynamic and embedded whole-body actions, where affect and affect attunement are "not enclosed in an inner mental sphere to be depicted from outside, but come into existence, change and circulate between self and other in the intercorporeal dialogue" (Fuchs \& De Jaegher, 2009, p. 479). Consequently, Fuchs and De Jaegher (2009) define the early mother-infant dialogue as a mutual incorporation. Throughout an individual's lifespan the innate attachment system is activated by external threats or internal sources of distress and, when it works appropriately, it leads to emotional security (Jurist, Slade, \& Bergner, 2008; Shaver \& Mikulincer, 2014). Positive affective states are conducive to exploration and learning, thus supporting the development of knowledge and skills. One's capacity to self-regulate affective states is a crucial part of effective and adaptive psychological functioning and coping (Larsen, 2000). In addition, "affective experience (especially valence, intensity, duration, and frequency), and the ability to self-regulate such experience, plays an important role in human social interaction, in particular when conditions are stressful" (Schore \& Schore, 2008, p. 129). Furthermore, individuals can also take action either to maintain or to change, i.e., to enhance or suppress the intensity of affect, or to prolong or shorten the affective episode (Larsen \& Prizmic, 2004). These capabilities are essential in human development and can be observed on multiple levels, including the physiological, cognitive, and interpersonal domains of an individual (Calkins \& Fox, 2002).

In summary, the aforementioned perspectives indicate that affect and the modulation of affect are multifaceted phenomena in their very nature. They are highly dynamic, interactional, embodied, and essential for human development and well-being. In this article, the term modulation of intensity of affects will preferably be used, to (1) acknowledge the processual and interactive character, and (2), to emphasize the idea of a sufficient modification of affective intensity within an interpersonal interaction, rather than an entire change from one unwanted affective state to another, more wanted one.

\section{THE MODULATION OF AFFECT IN MUSIC THERAPY}

Beside clinical applications across different cultures and continents, music serves as a self-regulative tool for many individuals in everyday life (DeNora, 2000; Uhlig, Jaschke, \& Scherder, 2013). Accordingly, many people use music -intentionally or habitually - to reduce and adapt to stress, to intensify emotions, or to enhance their well-being and health condition (Skån-
Exploring the modulation of affect in improvisational music therapy 
land, 2013; Sloboda, O’Neill, \& Ivaldi, 2001; van Goethem \& Sloboda, 2011; van Goethem, 2010). In music therapy, the expression, modulation and change of the intensity of affect in an interpersonal relationship is often a key element. Examples from clinical practice and research range from improvisational music therapy with children with autism (Nordoff \& Robbins, 1977/2007; Schumacher \& Calvet, 1999; Schumacher, Calvet, \& Reimer, 2011), and people with eating disorders (Trondalen \& Skårderud, 2007), to the use of familiar music with persons with dementia to meet their psychosocial needs and reduce agitation (Ridder, Stige, Qvale, \& Gold, 2013). In general, emotional and affective regulation in individuals with neurological conditions is a common challenge (Aldrigde, 2005). However, music therapy in intensive care and neurological rehabilitation focuses especially on the establishment of contact with people in a coma or persistent vegetative state, with music as an alternative medium for communication. Furthermore, music is applied to facilitate relaxation, or to create a familiar atmosphere (see e.g. Baker \& Tamplin, 2006; Kennelly \& Edwards, 1997; Tamplin, 2000, 2013). In practice, music therapists might sing familiar songs that have been emotionally meaningful for a person, or they might improvise music, related to an individual's breathing pattern (Gustorff \& Hannich, 2000; Jochims, 1990). Breathing patterns are and remain very individual in their tempo, rhythm, dynamics and expressivity in people in a coma. Their breathing can for example be flat, fleeting, anxious, vigorous, peaceful, or harsh. Even in breathing patterns of ventilated patients individual features can be identified (Gustorff \& Hannich, 2000). In the second vignette of this case study, an adapted form of this music therapy technique has been applied. The live improvised music was adjusted to the patient's breathing pattern, and the affective expression of his bodily-emotional situatedness. To improvise music in music therapy is one method, besides re-creating (i.e. performing pre-composed songs or musical pieces), composing, and listening to music (Bruscia, 2014). In improvisational music therapy the patient and therapist actively invent music together while playing, singing or moving (Schmid, 2015). In contrast, the music therapy approach applied in the first case vignette is music listening. Within this receptive experience, the patient listens to music (chosen by $\mathrm{him} /$ her and/or the therapist), and can respond to the experience silently, verbally or in another modality (for example movement, or drawings) (Bruscia, 2014).

\section{MARC}

Marc (his name has been changed) experienced a myocardial infarction at the age of 44 at his workplace followed by a hypo-ischemic brain injury, leav- ing him in a persistent vegetative state. After several months in intensive care and neurological rehabilitation, Marc was admitted to a home for the elderly, still presenting with extensive care needs. His wife and his parents, but also some of his friends, were very much taking care of him. He had visitors every day, creating a lively atmosphere in his room. Marc was referred for occupational and physiotherapy, and received nine sessions of individual music therapy altogether within the course of three months. One session lasted between 20 and 30 minutes. A colleague from occupational therapy had suggested music therapy for Marc with the idea and hope that music could reach him behind a wall of isolation.

In the following, two vignettes out of the first and second music therapy session with Marc will be presented. The two vignettes were chosen to illustrate and discuss two different approaches in music therapy with Marc: music listening in the first vignette, and musical improvisation in the second vignette. These two approaches vary greatly regarding Marc's level of involvement, self-activity, and possibilities for client-therapist interaction. While in the first vignette the music therapist sings pre-composed music for Marc that he might remember and be familiar with, the improvised music in the second vignette is conducted together with Marc, with both the music therapist and Marc contributing to the evolving music.

\section{FIRST CASE VIGNETTE}

In our first encounter Marc sat in a huge wheelchair with no recognizable voluntary movements, his eyes closed. Most notable was an unregulated affective state, expressed by a heavy, jerky breathing pattern and high body tension. Marc seemed to be extremely strained, and his wife, who was visiting him that day, told me that this was the way he typically was. His tracheostomy had remained in place because of his fragile and instable breathing condition. I asked his wife what Marc's involvement with music was before the incident, and she said that he had not played an instrument, but loved to listen to songs from Pink Floyd and Dire Straits. She agreed that I could document the music therapy sessions on video for later evaluation, and left the room. I sat opposite Marc, watching, listening, sensing. After a while, I started to sing "Swing Low, Sweet Chariot", with the idea that Marc might know the song, but also trying to create a calm and enclosing atmosphere. He continued with his heavy breathing, and seemed to be kept in a state of high arousal and restlessness. After a pause, I played the refrain of "Brothers in Arms" on the piano, introducing some music that Marc had had a relationship with before the incident, and with the intention to create a familiar atmosphere. Again I could not observe any response from him 
and felt very strongly that I was not able to reach him by making music for him. This led me to the idea of finally improvising on a kantele, a little string instrument, without addressing him or awaiting any reaction, but closing our first session with music. Later on I could see on the video, that Marc's heavy breathing led to minimal, involuntarily movement of his head to the left hand side. In reflection, these minimal movements might have been the reason for me to sing "Swing Low, Sweet Chariot" as a first song for Marc.

\section{SECOND CASE VIGNETTE}

In our second session one week later, Marc presented again with a high and unregulated level of affective expression and body tension. This time I decided to offer an improvisational music therapy approach, taking Marc's breathing movements as the starting point for joint music making. I responded to his breathing tempo with the tempo of my improvised music, and played some chords in a minor key on the piano, with the idea to cautiously combine movement and music. After a while I began to sing in a low register in an impulsive and gruff manner, trying to better match the expressive quality of his movements and bodily situatedness. While my intention in the beginning was to match Marc's movements as closely as possible with my music, allowing for the experience of sensory integration, I adapted my playing and singing to the dynamic affective qualities of his movements in the course of the improvisation. As I made myself more available to Marc on an emotional level and inner state of being, his affective expression could become audible and shared in the music. Within the further course of the improvisation, Marc suddenly stopped with his head movements, not moving at all for some seconds at the very first time. I stopped my singing and playing as well. This first pause was full of waiting, wondering, realizing that it was us doing that. After some seconds, Marc continued with his head movements, but intentionally changed their tempo and range. I changed the improvised music accordingly, reflecting his initiatives immediately, so that the music became lighter, with a swinging waltz movement. Compared to the very beginning of the improvisation Marc calmed down in the course of our joint music making, and seemed to be much more relaxed. After about five minutes he intentionally stopped with his movements once more, opened his eyes and looked directly at me. I stopped playing as well and the music came to an end.

Within this improvised musical encounter Marc could contribute with his movements and his affective expression to the extemporaneously joint music making. The music was flexibly created with him, and not for him, awaiting a certain reaction from him to my musical stimulus. The modulation of the intensity of his affect within improvised music was a core issue in the further course of the therapy. Attuning to Marc's movements and affective expression with my music led to a decrease in his body tension and intensity of breathing. These physiological changes could be observed in the following seven music therapy sessions as well. Unfortunately the therapy course came to an abrupt end after nine sessions, as Marc got severe pneumonia, and died unexpectedly some weeks later.

\section{DISCUSSION}

With a focus on the second case vignette out of music therapy with Marc, the role of affect modulation as a means of communication will be discussed with respect to embodiment approaches, expanding perspectives from developmental psychology and attachment theory presented earlier.

\section{MUSICAL FEATURES AS DYNAMIC FORMS IN AFFECT MODULATION}

The first case vignette illustrates a receptive and therapist-led music therapy approach, offering Marc to listen to calm music selected by me, assuming that he might remember and enjoy it. In contrast, in the second case vignette Marc's movements with their individual tempo and expressive qualities served as the starting point for the improvised music, allowing for sensory integration and affect attunement. In this way, both of us were contributing actively to the music: Marc with his movements and high, unregulated affect, and I with my singing and playing. Furthermore, Marc's affective expression with its intensity and vitality led the musical course, and by implication my playing and singing. I tried to attune to his affective expression as closely as possible. To approach and interact with another's internal state in this way has been termed affect attunement by the psychiatrist and psychoanalytic theorist Daniel Stern (1985/2000). Referring to Stern's concept, the music therapist Mercédès Pavlicevic (2002) suggests an approach to affect attunement in music therapy that she describes as dynamic forms, equating such forms to Stern's vitality affects $(1985 / 2000,2010)$. She argues that music provides a medium for reproducing properties that belong to feelings, movement, shape, contour, and intensity. In music therapy intersubjective musical and emotional relationships can develop in improvisations, with the client and therapist using similar dynamic forms in the modality of music. In this way, the expressive features of music, such as tempo, dynamics, timbre, and pitch, can correspond with the dynamic forms of affective, bodily-emotion-
Exploring the modulation of affect in improvisational music therapy 
al expression. These dynamic forms are expressed through the qualities of our acts. Consequently, it could be the dynamic forms of actions, their tempo, timbre or pitch, rather than the actions themselves, which enable individuals to interact with each other, and build up a relationship. In the context of our improvisation, Marc and I explored and played with the dynamic forms of his affective expression, their intensity, tenseness and restlessness, using the expressive features of music. Our playing with the affect was embodied in the musical form. It became a means of communication for the two of us beyond words and with Marc presenting the initiative and ownership in a way that had not been observed outside the music therapy context.

\section{AN EMBODIED PERSPECTIVE ON IMPROVISATIONAL MUSIC THERAPY}

Coming from an embodiment-informed perspective, Fuchs and De Jaegher (2009) suggest that interactions between individuals are enactive, dynamic and embedded whole-body actions. The coordinated moment-to-moment interaction of embodied agents and the agents' experience of this process emerge from the interactive practice and coordination of the persons involved: "Through the mutual coupling of their lived bodies - mediated through eye contact, facial expression, voice, touch, gesture and other kinds of intentional action - they enter a dyadic bodily state. Their body schemas and body experiences expand and, in a certain way, incorporate the perceived body of the other" (Fuchs \& De Jaegher, 2009, p. 472). Adapted to the second case vignette in music therapy with Marc, this could mean that within the intimate improvised moment-to-moment interaction both of us influenced each other with the expressive musical features and dynamic forms of our mutual activity, entering a dyadic bodily state. To make this happen, Marc contributed with his movements and affective expression to our intercorporeal dialogue, while I sang and played on the piano. Interestingly, I remember that I became more and more attentive in this encounter myself, presenting with increasing body tension. I was looking at Marc almost all the time, trying to couple and transform my visual and sensual observations and information directly into musical movement, through my singing and playing on the piano. I adapted with my body tension to his body tension, to become able to translate his state of being into musical expression. In this way, a mutual coupling of our lived bodies was mediated through sound, movement, and affective expression at the same time (Fuchs \& De Jaegher, 2009). We mutually influenced each other, creating and entering a dyadic bodily state within an affect-driven communication. Hence improvisational music therapy provides a multimodal, experiential field allowing for the application and interweaving of diverse modes of expression, impression and interaction. In the course of the improvisation, Marc and I utilized affective expression and modulation as a resource rather than an uncomfortable, stressful condition. Improvisational music therapy literally provides room for such enactive practices, determining the living body as a self-organizing system and its sense-making relation to the world (Schmid, 2013, 2015). Furthermore, this went hand in hand with physiological changes in Marc's condition. He became calmer and could gain control over his head movements, could breath more calmly and implement a new quality of relationship with another person. In a multilayered process, Marc's affect-laden, physiological and communicational conditions were intertwined, contributing to self-regulation (Porges, 2011). Taking an embodied perspective on music therapy with individuals not accessible by language-dependent approaches, the body with its posture, movements and gestures provides the vivid basis for human communication. Moreover, "through the medium of an active performed body, health is expressed and maintained" in individuals experiencing neurological trauma (Aldridge, 2005). Thus, a health-promoting therapeutic approach is largely about how a therapist is with a client. The neurologist Andreas Zieger invented the concept of a "körpernaher Dialog" (Zieger, 2002, p. 265) for being in dialogue with a person in a persistent vegetative state on a bodily-emotional level. In such dialogues, the semantic meaning of a body's posture, or gestures and movements, with their emotional and affective expression, form the nonverbal components for the interpersonal interaction.

\section{VIABILITY IN EMBODIED COMMUNICATION}

Considering the quality of these components, it is a fundamental prerequisite for every meaningful human communication and relationship that the information exchanged matches the situatedness and interest of the individuals involved (Schmid, 2013). It is the matching accuracy and meaningfulness, the viability of the information exchanged, that allows for interactional processes described in the second vignette, or that might prevent such processes as seen in the first case vignette. As with Marc, the more the dynamic forms of my response with their intensity, tempo, and timbre fitted to his expressive repertoire, the more he could come into exchange with me, in turn stimulating new responses in me. It was not words or gestures, but the dynamic forms (Pavlicevic, 2002) of our affective expression and modulation that served as the viable ingredients for Marc and me, interacting with each other intentionally on a non-verbal, highly meaningful level in the modality of music. 
It is a unique feature of music making that we can play and sing simultaneously or alternately. Like in early mother-infant interaction, both simultaneous and alternate modes of music making can facilitate contact and communication in music therapy with persons in extreme isolation due to neurological trauma. Affective states can become audible, visible and perceptible through instrumental play, singing, or moving, and thereby function as a form of social communication (see Larsen, 2000). In 2005, David Aldridge called neuro-degenerative diseases dialogue-degenerative diseases, pointing to the gradual loss of communicative possibilities for both individuals presenting neurological conditions and their social context. In this case study affect and the modulation of its intensity were discussed as multifaceted, embodied and intercorporeal events, deeply rooted in a human being. Music affects us and may evoke emotions, but even more important for music therapy, affective expressions can be shared. They can become a means of communication with very isolated people, limited in their expressive repertoire due to neurological trauma. Marc presented levels of activity, intentionality and interest in interpersonal contact in the improvised encounter that had not been observed outside music therapy. In music therapy, he could partly regain his interactive potential, and share his state of being within a communicative ecology, relieving his isolation for a period of time.

\section{CONCLUSIONS}

The main focus of this case study was to describe and explore affect modulation as a means of communication with Marc, a man experiencing extreme isolation due to neurological trauma. It might be a unique feature of improvisational music therapy to include affective expression and modulation of a patient as presented in this article. Within an improvisational music therapy approach, an individual's bodily-emotional situatedness and affective expression can work as the starting point for an enactive, non-verbal encounter. In this encounter a person can gain access to social and communicative capabilities, accompanied by positive physiological changes, improving his state of health and quality of life.

It is beyond the scope and intention of this case study to draw generalized conclusions. However, it is suggested to further explore and research affect modulation as a means of communication in music therapy with individuals living in a persistent vegetative state. This might contribute to an extended, specified repertoire of music therapy interventions for individuals in a persistent vegetative state and, furthermore, bring forth music therapy's particular contribution to interdisciplinary care and rehabilitation for this population.

\section{REFERENCES}

Aldridge, D. (ed.). (2005). Music Therapy and Neurological Rehabilitation. Performing Health. London: Jessica Kingsley Publishers.

Ammaniti, M., \& Gallese, V. (2014). The Birth of Intersubjectivity. Psychodynamics, Neurobiology, and the Self. New York: W.W. Norton \& Company.

Baker, F., \& Tamplin, J. (2006). Music therapy methods in neurorehabilitation. A clinician's manual. London: Jessica Kingsley.

Barrett, K. C., Fox, N. A., Morgan, G. A., Fidler, D. J., \& Daunhauer, L. A. (2013). Handbook of Self-Regulatory Processes in Development. New Directions and International Perspectives. New York and London: Psychology Press.

Bowlby, J. (1988). A secure base: Clinical applications of attachment theory. London: Routledge.

Bowlby, J. (1969/1982). Attachment and loss: Vol. 1. Attachment $\left(2^{\text {nd }}\right.$ ed.). New York: Basic Books.

Bruscia, K. E. (2014). Defining Music Therapy. (3 ${ }^{\text {rd }}$ ed.) University Park, IL: Barcelona Publishers.

Calkins, S. D., \& Fox, N. A. (2002). Self-regulatory processes in early personality development: A multilevel approach to the study of childhood social withdrawal and aggression. Development and Psychopathology, 14, 477-498.

DeNora, T. (2000). Music in everyday life. Cambridge: Cambridge University Press.

Fuchs, T., \& De Jaegher, H. (2009). Enactive intersubjectivity: Participatory sense-making and mutual incorporation. Phenomenology and the Cognitive Sciences, 8, 456-486. doi: 10.1007/s11097-009-9136-4

Gustorff, D., \& Hannich, H.-J. (2000). Jenseits des Wortes. Musiktherapie mit komatösen Patienten auf der Intensivstation [Beyond the word. Music therapy with coma patients in intensive care]. Bern: Hans Huber.

Jochims, S. (1990). Singend miteinander verbunden sein... Die Stimme im Zentrum der Therapie bei neurologischen Erkrankungen in der Frühphase [Be connected to each other in singing... The voice in the center of the treatment of neurological disorders in the early phase]. Musiktherapeutische Umschau, 11, 127-131.

Jurist, E. L., Slade, A., \& Bergner, S. (2008). Mind to Mind. Infant Research, Neuroscience, and Psychoanalysis. Other Press: New York.

Kennelly, J., \& Edwards, J. (1997). Providing music therapy to the unconscious child in the pediatric intensive care unit. Australian Journal of Music Therapy, 8, 18-29.

Larsen, R. J. (2000). Towards a science of mood regulation. Psychological Inquiry, 11, 129-141.

Larsen, R. J., \& Prizmic, Z. (2004). Affect regulation. In R. F. Baumeister \& K. D. Vohs (eds.), Handbook of self-regulation: Research, theory, and applications (pp. 40-61). New York: Guilford Press.
Exploring the modulation of affect in improvisational music therapy 
Mikulincer, M., Shaver, P. R., \& Pereg, D. (2003). Attachment Theory and Affect Regulation: The Dynamics, Development, and Cognitive Consequences of Attachment-Related Strategies. Motivation and Emotion, 27, 77-102.

Nordoff, P., \& Robbins, C. (1977/2007). Creative Music Therapy. New York, NY: Harper and Row.

Pavlicevic, M. (2002). Dynamic Interplay in Clinical Improvisation. Voices, 2.

Porges, S. W. (2011). The Polyvagal Theory: Neurophysiological Foundations of Emotions, Attachment, Communication, and Self-regulation. Norton Series on Interpersonal Neurobiology. New York: W. W. Norton \& Company.

Ridder, H. M., Stige, B., Qvale, L. G., \& Gold, C. (2013). Individual music therapy for agitation in dementia: an exploratory randomized controlled trial. Aging and Mental Health, 17, 667-678. doi: 10.1080/13607863.2013.790926

Schmid, W. (2015). A journey to memories - improvisational music therapy in neurological rehabilitation with a woman experiencing acquired brain injury (ABI). In C. Dileo (ed.), Case reports in advanced medical music therapy (pp. 232-238). Cherry Hill, NJ: Jeffrey Books.

Schmid, W. (2013). A penguin on the moon: Self-organizational processes in improvisational music therapy in neurological rehabilitation. Nordic Journal of Music Therapy, 23, 152-172. doi: 10.1080/08098131.2013.783096

Schumacher, K., Calvet, C., \& Reimer, S. (2011). Das EBQ-Instrument und seine entwicklungspsychlogischen Grundlagen [The AQR-instrument and it's developmental - psychologically foundations]. Göttingen: Vandenhoeck \& Ruprecht.

Schumacher, K., \& Calvet, C. (1999). The "AQR" - an Analysis System to Evaluate the Quality of Relationship during Music Therapy. Evaluation of interpersonal relationships through the use of instruments in music therapy with profoundly developmentally delayed patients. Nordic Journal of Music Therapy, 8, 188-191. doi: 10.1080/08098139909477974

Schore, J. R., \& Schore, A. N. (2008). Modern Attachment Theory: The Central Role of Affect Regulation in Development and Treatment. Clinical Social Work Journal, 36, 9-20. doi: 10.1007/s10615007-0111-7

Shaver, P. R., \& Mikulincer, M. (2014). Adult attachement and emotion regulation. In J. J. Gross (ed.), Handbook of Emotion Regulation ( $2^{\text {nd }}$ ed.) (pp 237250). New York, London: The Guildford Press.

Skånland, M. S. (2013). Everyday music listening and affect regulation: The role of MP3 players. International Journal of Qualitative Studies on Health and Well-being, 8, 20595. doi: 10.3402/qhw.v8i0.20595

Sloboda, J. A., O’Neill, S. A., \& Ivaldi, A. (2001). Functions of music in everyday life: An exploratory study using the Experience Sampling Method. Musicae Scientiae, 5, 9-32.

Stern, D. (1985/2000). The Interpersonal World of the Infant. A View from Psychoanalysis \& Developmental Psychology. New York: Basic Books.

Stern, D. (2010). Forms of Vitality: Exploring Dynamic Experience in Psychology, the Arts, Psychotherapy, and Development. Oxford, New York: Oxford University Press.

Tamplin, J. (2013). Adults in critical care. In J. Allen (ed.), Guidelines for Music Therapy Practice in Adult Medical Care. Gilsum, NH: Barcelona Publishers.

Tamplin, J. (2000). Improvisational music therapy approaches to coma arousal. Australian Journal of Music Therapy, 11, 38-51.

Trondalen, G., \& Skårderud, F. (2007). Playing with affects... and the importance of "affect attunement". Nordic Journal of Music Therapy, 16, 100111. doi: $10.1080 / 08098130709478180$

Uhlig, S., Jaschke, A., \& Scherder, E. (2013). Effects of music on emotion regulation: A systematic literature review. Proceedings of the $3^{\text {rd }}$ International Conference on Music \& Emotion (ICME3). G. Luck \& O. Brabant (eds.). Jyväskylä, Finland, 11-15 June 2013.

Van Goethem, A., \& Sloboda, J. (2011). The functions of music for affect regulation. Musicae Scientiae, 15, 208. doi: 10.1177/1029864911401174

Van Goethem, A. (2010). Affect regulation in everyday life: Strategies, tactics, and the role of music. (Unpublished doctoral dissertation). Keele University, Keele.

Zieger, A. (2002). Der neurologisch schwerstgeschädigte Patient im Spannungsfeld zwischen Bio- und Beziehungsmedizin [The neurologically severely injured patient in the tension between traditional and relational medicine]. Intensiv, 10, 261-274. 